\title{
XRD as an analytical tool for analyses of tommy atkins mango KERNEL polysAcharide
}

\author{
Cordeiro, E. M. S. ${ }^{1 *}$; Souza, N. F. ${ }^{2}$; Melo, E. F. ${ }^{2}$; Dias, A. F. ${ }^{3}$; Moraes, J. P. S. ${ }^{4}$; de \\ Souza Filho, M. M. ${ }^{4}$; Rosa, M. F. ${ }^{4}$; Ito, E. N. ${ }^{1}$ \\ 1 University Federal of Rio Grande do Norte-UFRN, Natal, Brazil \\ 2 University Federal of Ceará, Department Eng. Chemistry-UFC, Fortaleza, Brazil \\ 3 Institute Federal of Ceará, Department Process Chemistry-IFCE, Fortaleza, Brazil \\ ${ }^{4}$ Embrapa Agroindústria Tropical, Fortaleza, Brazil.
}

*ednamsc86@yahoo.com.br

\begin{abstract}
The aim of this work was to use X-Ray Diffraction (XRD) as an analytical tool for characterization of Tommy Atkins mango kernel starch and fibres. The experiments were performed in a Rigaku diffractometer DMAXB with $\mathrm{CuK} \alpha$ $(\lambda=1.5406 \AA)$ at $40 \mathrm{kV}$ and $40 \mathrm{~mA}$, analysis speed of $1^{\circ}(2 \theta) \cdot \mathrm{min}^{-1}$, with step scan of $0.05^{\circ}$, ranging between $2 \theta$ angle from $5^{\circ}$ to $40^{\circ}$ at $25^{\circ} \mathrm{C}$ to calculate Sega Crystallinity Index (CI). Mango starch presented diffraction peaks at $2 \theta=11.62^{\circ}, 15.12^{\circ}, 17.14^{\circ}$, and $23.1^{\circ}$, which represents a typical type A starch pattern. In this structure, amylopectin molecules are fitted to a monoclinic unit cell. Raw fibres presented peaks at $2 \theta=12^{\circ}$ and $22^{\circ}$, typical for a cellulose I pattern. Mercerized and bleached fibres, and nanowhiskers presented peaks at $2 \theta=15^{\circ}, 22^{\circ}$, and $34^{\circ}$, typical for cellulose II pattern. Cellulose I has two unit cell structures: $\mathrm{I}_{\mathrm{a}}$ (triclinic) and $\mathrm{I}_{\mathrm{b}}$ (monoclinic). Cellulose II occurs just as a monoclinic unit cell. The diffraction peaks of Cellulose II around $2 \theta=22^{\circ}$ are narrower and higher than in Cellulose I. Moreover, the chemical treatment washes off amorphous components of the fibre, remaining crystalline structures. It was possible calculate the CI for raw fibres $(29.4 \%)$, mercerized fibres $(50.3 \%)$, bleached fibres (69.8\%), and nanowhiskers (58.0\%). Thus, the XRD technique is an important analytical tool for identifies the crystalline pattern of the mango kernel polysaccharides, as well as to quantify the Segal Crystallinity Index.
\end{abstract}

